\title{
The Use of Nonlinear Digital Filtering in the Navigation Task of Integrated Inertial-Satellite System
}

\author{
I.V. Shcherban \\ Southern Federal University, ul. Mil'chakova 10 \\ Rostov-on-Don, 344090 Russia
}

\begin{abstract}
The tightly integration methods of inertial-satellite navigation systems (IS NS) based on estimation of dynamic error models of inertial navigation systems initially have a methodical errors caused by using the procedure of linearization of dynamic error models. This methodical errors are significantly increasing when the rough strapdown inertial navigation system (SINS) implemented based on technology of microelectromechanical systems using as the part of the IS NS. The method of integration of SINS measurements and satellite navigation systems measurements based on the nonlinear digital filtering has been given. This method would eliminate the loss of non-linear relationships in the construction of optimal estimation procedures of navigation parameters in IS NS.
\end{abstract}

Keywords- Integrated inertial-satellite navigation system; rough strap down inertial navigation system; micro electromechanical systems; nonlinear digital filtering

\section{INTRODUCTION}

The traditional methods of tightly integration of inertial-satellite navigation systems (IS NS) initially have a methodical error caused by linearization of dynamic error models of inertial navigation systems and measurement models of satellite navigation systems (SNS) [1,2]. Ability to perform linearization procedure is justified by assumption that the deviation of measurement errors from their model values is small. When solving the dynamic equations of errors of inertial navigation systems, which are the variational equations, methodical errors occur inevitably. These errors result from the assumption about the smallness of the measurement errors of inertial sensors and from procedures of linearization of the model equations $[1,3,4]$.

At the same time, when using the rough strapdown inertial navigation system (SINS) implemented based on technology of microelectromechanical systems (MEMS) as the part of IN SS, such an assumption is unacceptable and causes a significant increase in the above-mentioned methodical errors [5]. This is explained by the fact that, due to an unstable power supply and temperature factor the error models of MEMS-sensors are characterized by uncontrolled nonstationarity behavior of parameters. In tightly associated integrated navigation systems such an a-priori unknown increase of methodical linearization error, for example, determines the known problems with convergence of complicated stochastic filters [1-5].

Therefore, there is a need for a method of integration of SINS measurements and SNS measurements that would eliminate the loss of non-linear relationships in the construction of optimal estimation procedures of navigation parameters in IS NS.

Similar problems arise in the construction of small-sized mobile inertial-satellite navigation systems used as a part of control systems of unmanned aircrafts or motor vehicles.

\section{The Object And The Observer Models Described BY THE STATE-SPACE REPRESENTATION}

In order to overcome the above mentioned problem, we propose to solve it by applying the theory of nonlinear stochastic filtering and, therefore, using the statistically optimal digital filter. For the synthesis of such a filter the following complete nonlinear model of SINS described by the state-space representation is needed:

$$
\dot{Y}=F(Y, t)+F_{0}(Y, t) \xi(t),
$$

where $Y(t)$ - a state vector of SINS; $\xi(t)$ - a white Gaussian noise (WGN) vector with known parameters; $F(Y, t), F_{0}(Y, t)$ - known nonlinear vector and matrix functions of the corresponding dimensions.

The similar model is presented in the work [6], where $Y=\left|\begin{array}{llllllll}\lambda & \varphi & h & V_{E} & V_{N} & V_{h} & M_{\mathrm{CE}}^{(\mathrm{v})}\end{array}\right|^{\mathrm{T}}-$ is a vector of the dimension $15 \times 1,{ }^{\mathrm{T}}$ - the sign of transposition operation, $\varphi, \lambda, h$ - geodetic latitude, longitude and height, respectively; $V_{E}, V_{N}, V_{h}$ - east, north and vertical components of the linear velocity relative to the Earth SINS; $M_{C E}{ }^{(\nu)}$ - vector composed of the components of the Poisson matrix ( $\operatorname{dim}\left(M_{C E}\right)=3 \times 3$ ), and functions dimension are $16 \times 1,15 \times 1$ and $15 \times 6$, respectively. The choice of this particular nonlinear dynamic model is explained by the fact that it can be used to describe the MEMS SINS with different sets of sensors and allows the realization of methods of nonlinear digital filtering for integration of the heterogeneous measurement information in the IS NS [6].

To synthesize the filter the external to the model (1) observer, the model of which must also be submitted in the form of state space, is required. Given that the available output of the majority of mobile on-board navigation receivers SNS are the geographical coordinates of the current position of the object and the absolute value of its absolute velocity $V_{m}$, the 
model "observer" for "object" (1) has the following canonical representation:

$$
Z=H(Y, t)+\eta(t),
$$

Where $\quad Z=\left|\begin{array}{llll}\lambda & \varphi & V_{m}\end{array}\right|^{\mathrm{T}} \quad-$ is a vector of measurements with the dimension $3 \times 1 ; \eta(t)-$ WGN vector with variance matrix $D_{\eta}(t)$; $\boldsymbol{H}(Y, t)=\left|\begin{array}{lll}1 & 1 & \sqrt{{V_{E}}^{2}+V_{N}{ }^{2}+V_{h}{ }^{2}}\end{array}\right|^{\mathrm{T}}$. The model (2) is written in a generalized form according to the assumption about the random nature of such basic errors of satellite measurements, as a receiver clock shift relative to the system scale, ionospheric and tropospheric signal delay on the propagation path, the hardware errors of the vehicle-borne receiver, error due to multipath [1-4].

Equations (1), (2), represented in the classical "object-observer" form allow a theoretically rigorous a-posteriori optimal nonlinear estimation of the SINS state vector with the selected probabilistic criterion. Since the exact solution of the problem is related to the need to integrate the integro-differential equations in partial derivatives (Stratonovich equation) for the a-posterior probability density function (APDF), then in order to to reduce the computational cost, the suboptimal estimation of the navigation vector based on nonlinear local Gaussian approximation APDF is used [3].

\section{Algorithm FOR NONLINEAR Estimation OF THE STATE VECTOR SiNS}

The SNS navigation messages are formed in general case in non-equidistant instants of time $t_{j-1}, t_{j}, t_{j+1}$, , where $j$ - is the number of reception cycle of the navigation message [1-3]. The estimation of the current navigation parameters is done from measurements of SINS in the predictor block at $T=t_{j}-t_{j-1}$ time intervals between discrete satellite measurements. Continuous (high-frequency) estimation based on navigation task solution and derived according to the information from SINS is executed at a rate $T_{0}<<T$, determined by the performance of the onboard calculator, during $k=T / T_{0}$ iterative cycles. As a result, the vector of navigation parameters $Y(k, j)$ is calculated in the final k-th cycle of the predictor block.

In the correction block the discrete non-linear Gaussian filter is used at the time instant $t=t_{j}$ [3]. Therefore, initial condition for corrections and write-offs of SINS errors here is the vector $Y(k, j)$ of navigation parameters, denoted in [3] by $\hat{Y}\left(t_{j}-0\right)$. Such a relation between the initial and final conditions of the discrete filtering in the proofreading block and the continuous estimation in the predictor block is one of the main conditions for the close integration of measurements of the autonomous MEMS SINS and measurements SNS [3].

For calculating the measurement model (2) navigation messages SNS with $T$ discretization are used. Hence, for the $j$-th navigation message the vector-function $H$ in equation (2) is calculated from the determined state vector $\hat{Y}\left(t_{j}-0\right)$. Therefore, the measurement model (2) can be represented in a discrete form as

$$
Z_{j}=H\left(\hat{Y}\left(t_{j}-0\right), t_{j}\right)+\eta\left(t_{j}\right) .
$$

The equations of nonlinear discrete Gaussian filter [3] are of the form:

$$
\begin{array}{r}
\hat{Y}\left(t_{j}+0\right)=\hat{Y}\left(t_{j}-0\right)+P\left(t_{j}+0\right) \frac{\partial H\left(\hat{Y}\left(t_{j}-0\right), t_{j}\right)}{\partial \hat{Y}^{\tau}} D_{\eta}{ }^{-1}\left[Z_{j}{ }^{n}-H\left(\hat{Y}\left(t_{j}-0\right), t_{j}\right)\right] \\
P^{-1}\left(t_{j}+0\right)=P^{-1}\left(t_{j}-0\right)+\left[\frac{\partial H\left(\hat{Y}\left(t_{j}-0\right), t_{j}\right)}{\partial \hat{Y}^{T}}\right)^{T} D_{\eta}{ }^{-1} \frac{\partial H\left(\hat{Y}\left(t_{j}-0\right), t_{j}\right)}{\partial \hat{Y}^{T}}
\end{array}
$$

where $Z_{j}{ }^{\text {и }}$ is the measurement vector formed from the $\mathrm{j}$-th navigation message SNS.

Optimal estimations of the state vector SINS $\hat{Y}$ are formed as the result of solving of equations (4) according to the information from the $j$-th navigation message SNS.

\section{CONCLUSION}

The numerical investigations have confirmed the correctness of the devised algorithm. Due to the consistent application of "prediction" procedures based on continuous direct solution of the problem of inertial navigation and "correction" based on the discrete nonlinear filtering, it was possible to ensure tight integration of MEMS SINS and SNS without methodical errors caused by linearization of models. Thus, the proposed algorithm has no problems with convergence of the filter.

The developed algorithm can be used in the construction of the small-size mobile integrated inertial-satellite navigation systems in unmanned aircrafts or motor vehicles. (RFMEFI59414X0002).

\section{REFERENCES}

[1] A.A. Golovan, N.A. Parusnikov, Matematicheskie osnovy navigatsionnykh sistem. Ch. I. Matematicheskie modeli inertsial'noi navigatsii (Mathematical Fundamentals of Navigation Systems. Ch. I. Mathematical Models of Inertial Navigation), second ed., Mos. State Univ., Moscow, 2010.

[2] M.S. Grewal, A.P. Andrews, Application of Kalman Filtering to GPS, INS and Navigation (Notes), CA: Kalman Filtering Consulting, Anaheim, 2000.

[3] A.I. Perov, Kharisov V.N., GLONASS. Printsipy postroeniya i funktsionirovaniya (GLONASS. Principles of Construction and Functioning), Radiotekhnika, Moscow, 2010.

[4] O.V. Demidov, Problem of cramped integration of GLONASS and GPS systems with INS of different classes of exactness, PhD. Sci. (Phys.-Math.) Dissertation, Mos. State Univ., Moscow, 2009.

[5] V.J. Raspopov, V.V. Matveev, Osnovy postroenya besplatformennyh inerthialnyh navigathionnyh system (The Fundamental Principles of the Development of Strapdown Inertial Navigation Systems), Eletropribor, Saint Petersburg, 2009. 
[6] I.V. Shcherban, S. A. Tolmachev Universal Model of a Ground Vehicle for Realization of Integrated Inertial-Satellite Navigation Systems, Automatic Control and Computer Sciences, 2014, vol. 48, No. 3, pp. 159-166. 